\title{
8.4 Novel autoantibodies targeting a p I 40 protein are a major autoantigen system in juvenile dermatomyositis and a marker of calcinosis
}

\author{
H Gunawardena*1, LR Wedderburn ${ }^{2}$, ZE Betteridge 3 , H Chinoy ${ }^{4}$, J North ${ }^{3}$, \\ RG Cooper ${ }^{5}$, AV Ramanan ${ }^{6}$, JE Davidson ${ }^{7}$ and NJ McHugh ${ }^{1}$
}

\begin{abstract}
Address: ${ }^{1}$ Rheumatology Department, Royal National Hospital for Rheumatic Diseases, Bath, UK, ${ }^{2}$ Rheumatology Unit, Institute of Child Health, London, UK, ${ }^{3}$ School for Health, University of Bath, Bath, UK, ${ }^{4}$ Centre for Integrated Genomic Medical Research, University of Manchester, Manchester, UK, ${ }^{5}$ Rheumatic Diseases Centre, Hope Hospital, Salford, UK, ${ }^{6}$ Rheumatology Department, Bristol Royal Hospital for Children, Bristol, UK and ${ }^{7}$ Rheumatology Department, Royal Liverpool Children's Hospital, Liverpool, UK

* Corresponding author
\end{abstract}

from I5th Paediatric Rheumatology European Society (PreS) Congress

London, UK. 14-17 September 2008

Published: 15 September 2008

Pediatric Rheumatology 2008, 6(SuppI I):SI8 doi:I0.II86/I546-0096-6-SI-SI8

This abstract is available from: http://www.ped-rheum.com/content/6/SI/SI8

(c) 2008 Gunawardena et al; licensee BioMed Central Ltd.

\section{Background}

To demonstrate that autoantibodies targeting a p140 protein are a major autoantigen in juvenile dermatomyositis (JDM) and describe the clinical associations in children recruited to the JDM Registry (JDRR).

\section{Methods}

156 children were studied. Serum was screened by immunofluorescence (IF) and radio-immunoprecipitation (IPP) [1]. Immunodepletion was used to establish whether p140 is different to p155/140 also recognised in JDM [1].

\section{Results}

$21 \%$ of children were positive for anti-p140 on IPP, with a weak non-specific nuclear pattern or negative ANA on IF. No anti-p140 cases were positive for other autoantibody specificities. Immunodepletion confirmed that p140 and p155/140 are different autoantigens. Anti-p140 positives compared to negatives had a similar male:female ratio and age at diagnosis. No significant difference was observed in the type or distribution of rash when comparing anti-p140 positives vs. negatives except for more rash on the trunk in negative cases $(\mathrm{p}=0.017)$. Calcinosis was significantly more frequent in anti-p140 positives $(52 \%)$ compared to negatives $(13 \%)$ ( $p<0.001$, OR $7.195 \%$ CI
3-16.8). When comparing anti-p140 and anti-p155/140 cases; cutaneous oedema $(p=0.04)$ and rash over the trunk $(\mathrm{p}=0.002)$ and small joints $(\mathrm{p}=0.013)$ was more frequent in anti-p155/140. Calcinosis in anti-p140 remained a significant feature compared to anti-p155/140 ( $\mathrm{p}=0.005$, OR $6.495 \%$ CI 2-22).

\section{Conclusion}

Anti-p140 found in this cohort is likely to be the same as anti-MJ, described against nuclear matrix protein NXP-2 [2]; further confirmation is required. Anti-p140 is a major autoantibody subset in JDM. Further characterisation of this system will provide insights into the pathophysiology of calcinosis in JDM.

\section{References}

I. Gunawardena H, Wedderburn LR, North J, Betteridge ZE, Dunphy J, Chinoy H, Davidson JE, Cooper RG, McHugh NJ, for the Juvenile Dermatomyositis Research Group UK: Clinical associations of autoantibodies to a p/55//40 kDa doublet in juvenile dermatomyositis. Rheumatology 2008, 47:324-8.

2. Targoff IN, Trieu EP, Levy-Neto M, Fertig N, Oddis CV: Sera with Autoantibodies to the MJ antigen react with NXP2. Arthritis Rheum 2007, 56(Suppl 9):S787. 